\title{
铁掺杂羟基磷灰石的制备及在强磁场中的定向研究
}

\author{
王小龙 ${ }^{1,2}$, 任忠鸣 ${ }^{1}$, 常 江 $^{2}$
}

(1. 上海大学 省部共建高品质特殊钢冶金与制备国家重点实验室, 上海市钢铁冶金新技术开发应用重点实验室, 材料科学与工程学院, 上海 200072; 2. 中国科学院 上海硅酸盐研究所, 上海 200050)

摘 要: 生物陶瓷表面纳米结构能够影响成骨细胞增殖和分化, 其表面微观结构的控制, 特别是烧结前晶粒取向调 控, 是设计开发生物活性陶瓷的关键之一。针对羟基磷灰石晶粒取向调控问题, 重点研究了铁掺杂羟基磷灰石晶体 在强磁场中的取向。分别采用共沉淀法和共沉淀-水热法合成了羟基磷灰石(HA)和铁-羟基磷灰石(Fe-HA), 通过 XRD、SEM、TEM、PPMS 和 ICP 等对 HA 和 Fe-HA 的物相、微观形貌、磁学性能、元素组成进行了表征和分析。 研究发现: Fe-HA 物相与 HA 相同, 没有明显的杂质相; HA 为抗磁性, Fe-HA 转化为顺磁性; 共沉淀法粉体为针状, 共沉淀一水热法粉体为短棒状, 针状粉体在强磁场中不能定向, 短棒状粉体能够定向; 在单一方向强磁场中, HA 不 能单轴定向, Fe-HA 能够在一定程度上沿 $c$ 轴取向。

关 键 词: 铁-着基磷灰石; 强磁场; 顺磁性; 定向

中图分类号: TQ174 文献标识码: A

\section{Synthesis and Orientation of Fe-doped Hydroxyapatite in High Magnetic Field}

\author{
WANG Xiao-Long ${ }^{1,2}$, REN Zhong-Ming ${ }^{1}$, CHANG Jiang ${ }^{2}$
}

(1. State Key Laboratory of Advanced Special Steel, Shanghai Key Laboratory of Advanced Ferrometallurgy, School of Materials Science and Engineering, Shanghai University, Shanghai 200072, China; 2. Shanghai Institute of Ceramics, Chinese Academy of Sciences, Shanghai 200050, China)

\begin{abstract}
The surface nanostructure of bioceramics can affect osteoblast proliferation and differentiation, therefore the control of the surface microstructure of bioceramics, especially grain orientation before the ceramic sintering, is one of key to design and develop bioactive ceramics for bone regeneration. This study aimed to investigate the crystal orientation of Fe-doped hydroxyapatite in high magnetic field. Pure hydroxyapatite (HA) and Fe-doped hydroxyapatite (Fe-HA) powders were prepared by co-precipitation method and co-precipitation-hydrothermal method, and their phase composition, microstructure, magnetic property, and elementary composition were analyzed by XRD, SEM, TEM, PPMS, and ICP. The results showed that the phase of Fe-HA is the same as HA with no impurity phase. HA is diamagnetic originally, while Fe-HA becomes paramagnetic. The microstructure of samples prepared by co-precipitation method is needle cluster-like but that prepared by co-precipitation-hydrothermal method is rod-like. It is the rad-like but not the needle cluster-like Fe-HA can be orientated in high magnetic field. Therefore, the orientation of rod-like Fe-HA can be regulated by high magnetic field. Furthermore, pure HA cannot be uniaxial orientated in single-directed high magnetic field. while Fe-HA can be oriented along $c$ axis in certain degree in singledirected high magnetic field.
\end{abstract}

Key words: Fe-HA; high magnetic field; paramagnetic; orientate 
羟基磷灰石(Hydroxyapatite, HA) 是人体骨骼和 牙齿的主要无机成份, 具有良好的生物相容性, 在 人体骨组织修复中被广泛使用 ${ }^{[1]}$ 。近年来有研究显 示, 纳米结构的差基磷灰石能够有效地促进细胞粘 附、增殖、分化和骨整合 ${ }^{[2-3]}$, 并且羟基磷灰石不同 晶面对于细胞行为以及蛋白质的吸附有选择性 ${ }^{[4]}$ 。 因此, 制备具有晶粒定向排列的羟基磷灰石陶瓷, 有助于揭示羟基磷灰石对于细胞行为和蛋白质选择 吸附的作用机理, 开发高性能骨修复材料。

随着近年来强磁场技术的发展, 非铁磁性材料 在强磁场中的定向受到越来越多的关注。原本可以 被忽略的弱磁性晶体的磁各向异性, 在强磁场中会 变得显著, 晶体在磁场中会发生取向, 使得晶体的 磁各向异性能降到最小, 以达到稳定状态。羟基磷 灰石为抗磁性晶体, 六方晶形。 $a 、 b$ 轴磁化率相同, 与 $c$ 轴磁化率不同, 为在强磁场中的定向提供了条 件。由于羟基磷灰石的固有特性, 单一方向外强磁 场并不能使差基磷灰石晶粒进行定向排列 ${ }^{[5]}$ 。有文 献报道, 在水平旋转强磁场中注浆成型羟基磷灰石 能够获得具有一定定向度的羟基磷灰石陶瓷 ${ }^{[6-7]}$, 但这种特殊的装置造价高, 在国内还鲜有相关报道。

对差基磷灰石进行离子掺杂改性, 改变羟基磷 灰石的理化性质, 特别是加入铁离子, 能够改变羟 基磷灰石的磁学性能 ${ }^{[8-11]}$ 。但对羟基磷灰石的铁离 子改性目前还主要是为了研究羟基磷灰石的磁热性 能以用于肿瘤热疗, 对其在强磁场中的定向效果还 没有相关文献报道 ${ }^{[12]}$ 。本研究在羟基磷灰石合成过 程中对其进行铁离子掺杂改性, 并研究铁离子改性 后的差基磷灰石在单一方向外强磁场中的定向效果 及规律, 并对其定向机理进行初步讨论。

\section{1 实验方法}

实验所使用的四水硝酸钲 $\left(\mathrm{Ca}\left(\mathrm{NO}_{3}\right)_{2} \cdot 4 \mathrm{H}_{2} \mathrm{O}\right)$, 磷 酸氢二胺 $\left(\left(\mathrm{NH}_{4}\right)_{2} \mathrm{HPO}_{4}\right)$, 三氯化铁 $\left(\mathrm{FeCl}_{3}\right)$, 十六烷 基三甲基溴化铵(CTAB), 十二烷基硫酸钠(SDS), 聚乙二醇 400(PEG), 氨水和无水乙醇统一购置于国 药集团化学试剂有限公司, 分析纯。

\section{1 材料的合成与制备}

(a)共沉淀法制备粉体

分别配制等体积 $0.5 \mathrm{~mol} / \mathrm{L}$ 的 $\mathrm{Ca}\left(\mathrm{NO}_{3}\right)_{2} \cdot 4 \mathrm{H}_{2} \mathrm{O}$ 溶 液和 $0.3 \mathrm{~mol} / \mathrm{L}$ 的 $\left(\mathrm{NH}_{4}\right)_{2} \mathrm{HPO}_{4}$ 溶液, 在 $\mathrm{Ca}\left(\mathrm{NO}_{3}\right)_{2} \cdot 4 \mathrm{H}_{2} \mathrm{O}$ 溶液中加入摩尔分数为 $1 \% 、 2 \% 、 5 \%$ 的 $\mathrm{FeCl}_{3}$ 以及 一定量的 CTAB 作分散剂。将 $\left(\mathrm{NH}_{4}\right)_{2} \mathrm{HPO}_{4}$ 溶液滴加 入 $\mathrm{Ca}\left(\mathrm{NO}_{3}\right)_{2} \cdot 4 \mathrm{H}_{2} \mathrm{O}$ 溶液, 持续搅拌, 使用氨水调节 $\mathrm{pH}$, 保持反应过程中 $\mathrm{pH}=11.0$, 反应 $24 \mathrm{~h}$, 使用去离
子水清洗 3 遍, 无水乙醇清洗 3 遍, 干燥后得到共沉 淀法粉体。

(b)共沉淀水热法制备粉体

使用上述共沉淀法得到羟基磷灰石沉淀液, 随 后将沉淀液转移到水热釜中, $150^{\circ} \mathrm{C}$ 反应 $3 \mathrm{~h}$, 反应 后, 使用去离子水清洗 3 遍, 无水乙醇清洗 3 遍, 干 燥后得到共沉淀水热法粉体。

(c)粉体在强磁场中注浆成型

取一定量粉体分别加入 $5 \mathrm{wt} \%$ 的 SDS、PEG 和 CTAB 作分散剂配制成浆料, 在强磁场中注浆成型。

\section{2 表征方法}

采用 $\mathrm{X}$ 射线衍射仪(Bruker D8 DISCOVER)表 征铁-羟基磷灰石粉体的相组成以及素坏的微区定 向度; 通过综合物性测试系统(PPMS Dynacool)研 究粉体的磁学性能; 将铁-羟基磷灰石粉体溶于稀 盐酸, 使用 715-ES 电感耦合等离子体原子发射光谱 仪研究粉体的元素组成; 通过场发射扫描电子显微 镜 (SU8220) 和场发射透射电子显微镜 (JEM-2100F) 观察粉体微观形貌。

\section{2 结果与讨论}

\section{1 物相分析}

采用共沉淀法和共沉淀-水热法合成 Fe-HA 粉 体的 XRD 结果见图 1 。

根据文献调研, 本实验选取 Fe 离子含量分别为 $1 \mathrm{~mol} \%, 2 \mathrm{~mol} \%, 5 \mathrm{~mol} \%$ 和 $10 \mathrm{~mol} \%$ (预实验发现，当 Fe 离子浓度为 $10 \mathrm{~mol} \%$ 时, 产物中出现较明显杂质 相)。共沉淀法合成 Fe-HA 粉体结晶度较低(图 1(a)), 共沉淀一水热法合成 Fe-HA 粉体结晶度比较高(图 1(b))。通过对比 XRD 图谱与 HA 标准卡片(09-0432) 发现, 共沉淀法合成 Fe-HA 与共沉淀一水热法合成 Fe-HA 样品都具有纯 HA 相, 没有出现其他杂质相。 因此, 选取 5 mol\%Fe-HA 进行后续的实验。

\section{2 磁学性能分析}

对合成的 HA 和 5mol\%Fe-HA 磁学性能进行表 征, 其磁化曲线如图 2 所示。可以看出, 不加入 $\mathrm{Fe}^{3+}$ 的 HA 磁化曲线为斜率为负的近直线, 说明 HA 原 本是具有抗磁性的; 在加入 $5 \mathrm{~mol} \%$ 的 $\mathrm{Fe}^{3+}$ 以后, 其 磁化曲线变为斜率为正的近直线, 说明 $\mathrm{HA}$ 由原本 的抗磁性转变为顺磁性, HA 磁学性能发生了顺磁 改变。

\section{3 元素组成分析}

由 XRD 结果可以看出, 掺杂后样品具有纯 HA 相, 后续实验选取 $5 \mathrm{~mol} \% \mathrm{Fe}$ 离子掺杂量对粉体的 定向效果进行研究。使用 ICP-OES 测量不同批次 

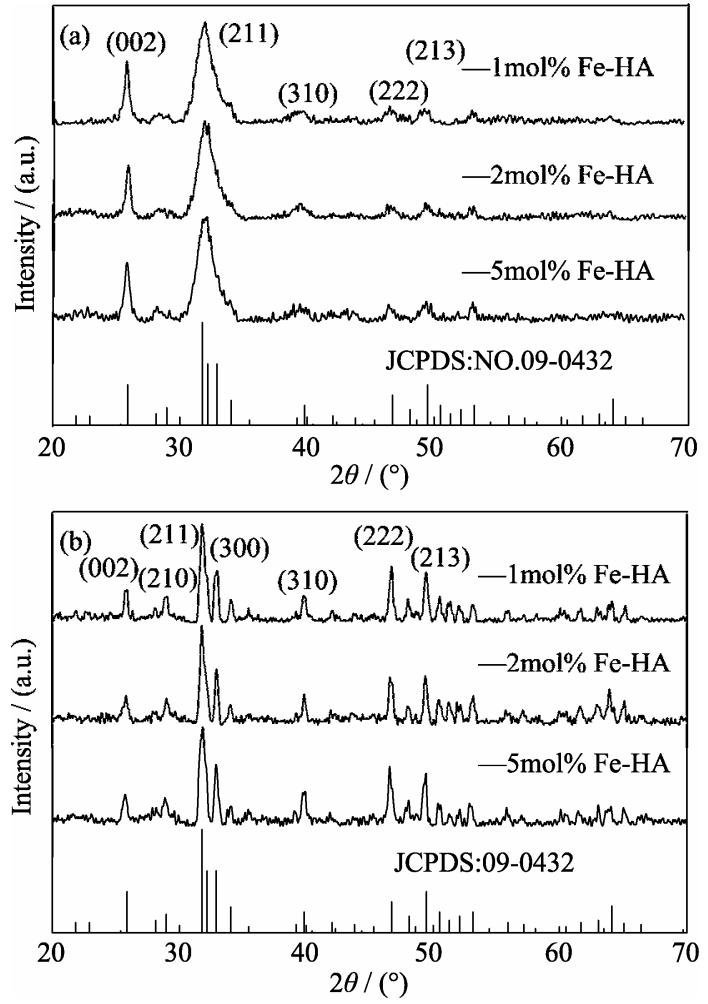

图 1 添加 $1 \mathrm{~mol} \% 、 2 \mathrm{~mol} \%$ 和 $5 \mathrm{~mol} \%$ 铁离子样品的 XRD 图谱 Fig. 1 XRD patterns of the samples doped with $1 \mathrm{~mol} \%$, $2 \mathrm{~mol} \%$, 5mol\% Fe ion

(a) Co-precipitation; (b) Coprecipitation-hydrothermal
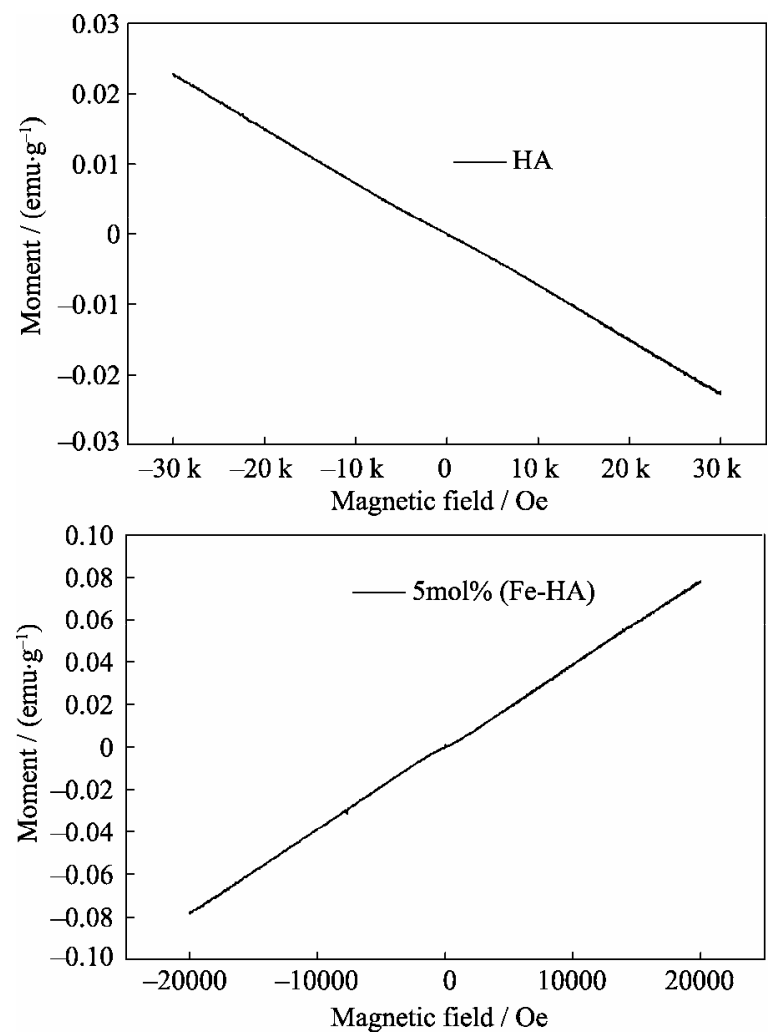

图 2 样品的磁化曲线 $\left(\mathrm{Oe}=\frac{10^{3}}{4 \pi} \mathrm{A} / \mathrm{m}, \mathrm{emu} / \mathrm{g}=4 \pi \times 10^{-7} \mathrm{~Wb} \cdot \mathrm{m} / \mathrm{kg}\right)$

Fig. $2 \quad B$ - $H$ curves of sample
$5 \mathrm{~mol} \% \mathrm{Fe}-\mathrm{HA}$ 的元素组成结果如表 1 所示。

$\mathrm{HA}$ 的钻磷比为 1.67 , 但加入 $\mathrm{Fe}^{3+}$ 以后, 样品的 铻磷比为 1.58 , 而 (钙 + 铁)与磷的比值大约为 1.67 , 这与文献报道是一致的 ${ }^{[8]}$, 铁在合成过程中可能部 分取代了羟基磷灰石中的钙离子。

\section{4 粉体微观结构分析}

采用扫描电镜和透射电镜对共沉淀法合成的 Fe-HA、共沉淀-水热法合成的 Fe-HA 和共沉淀-水 热法合成的 HA 微观形貌进行观察。

样品的 SEM 照片如图 3 所示, 共沉淀法制得的 Fe-HA 的微观形貌为针状, 并且晶粒不是单分散形式 存在, 而是以随机方式组合成针簇状(图 3(a)); 相比 于共沉淀法的 Fe-HA, 共沉淀-水热法制得的 Fe-HA 和 HA 经水热处理后, 微观形貌均由针状结构变为短 棒状结构, 长度在 $100 \mathrm{~nm}$ 左右, 并且不再为族状形 态, 而是变为单分散纳米棒结构(图 3(b), (c))。

样品的 TEM 照片如图 4 所示。共沉淀制备的 HA 和 Fe-HA, 颗粒长度为 50 100 nm, 结晶度较低, 几个颗粒堆积在一起形成单体(图 4(a), (b)); 共沉淀一 水热法制备的 HA 和 Fe-HA, 在水热处理后晶体结 晶度明显提升, 呈现出六棱柱状晶体结构, 并且相 比于共沉淀粉体来说, 分散性也明显提升, 呈现出 单分散性(图 4(c), (d))。

从 XRD、SEM 和 TEM 结果可以看出, 共沉淀 法制备的粉体结晶度比较低, 晶体表面的自由能较 高, 在晶体生长过程中, 相邻晶体会通过相互结合 来降低整体的自由能; 共沉淀一水热法制备的粉体, 结晶度比较高, 晶体表面的自由能相对较低, 晶体 不易发生相互聚集。

\section{5 定向度分析}

$\mathrm{HA}$ 的定向度常用 $R\left(R=I_{(002)} / I_{(211)}\right)$ 值评判 ${ }^{[13-15]}$, 即用样品(002)衍射峰强度除以(211)衍射峰强度。共 沉淀一水热法 Fe-HA 使用不同分散剂的浆体在垂直 方向强度为 $5 \mathrm{~T}$ 的强磁场中注浆成型的样品 XRD 结 果如图 5 所示。

一般情况下, HA 的 $\mathrm{R}$ 值为 $0.3 \sim 0.4$, 未加入分散 剂浆体注浆后样品的 $R=0.45$, 相比于普通 $\mathrm{HA}, R$ 值 略有增大, 加入 SDS 作为分散剂后, 样品的 $R$ 值有

表 14 组 5mol\%Fe-HA 样品的元素组成 Table 1 Elemental composition of four kinds of samples

\begin{tabular}{cccc}
\hline Sample & $\mathrm{Fe} / \mathrm{Ca}$ & $\mathrm{Ca} / \mathrm{P}$ & $(\mathrm{Ca}+\mathrm{Fe}) / \mathrm{P}$ \\
\hline S1 & 0.050 & 1.561 & 1.639 \\
S2 & 0.052 & 1.580 & 1.663 \\
S3 & 0.052 & 1.583 & 1.665 \\
S4 & 0.051 & 1.589 & 1.669 \\
\hline
\end{tabular}



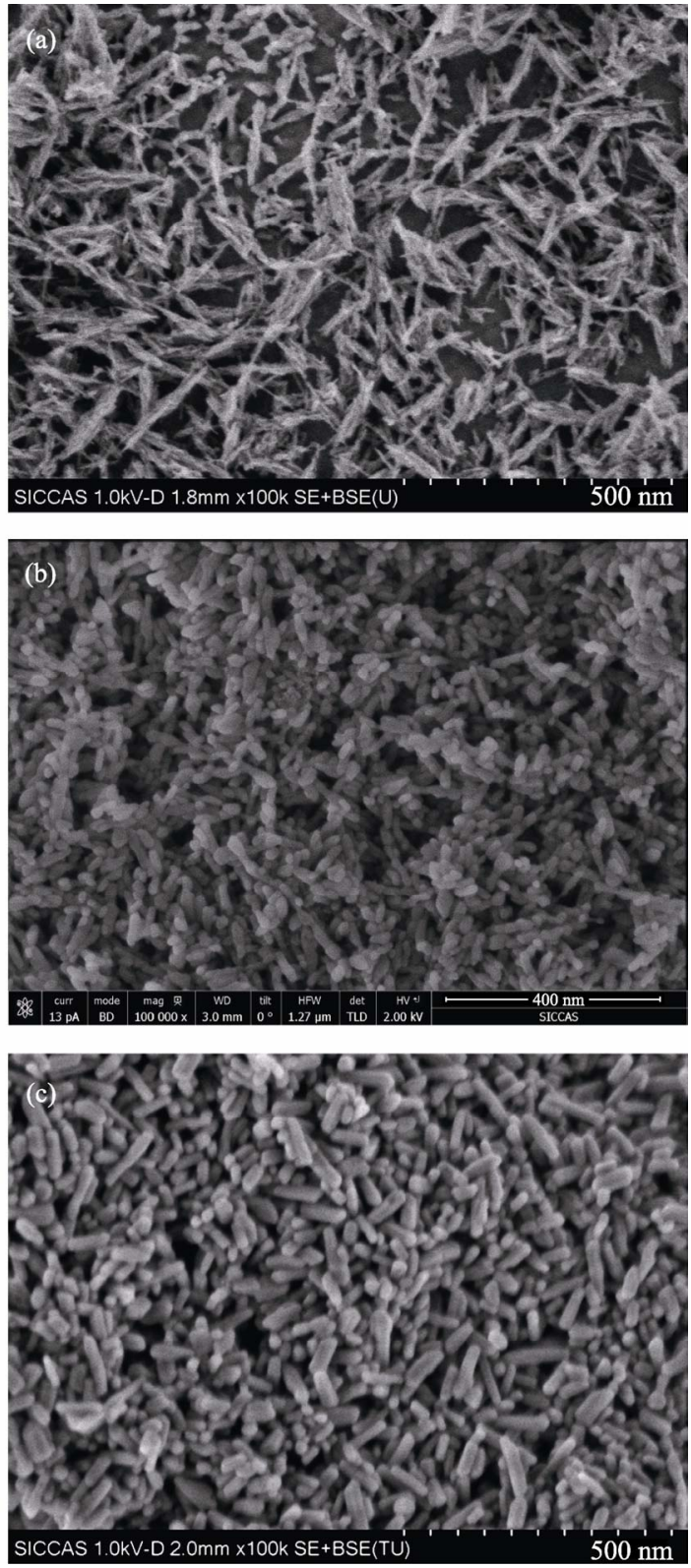

图 3 Fe-HA 和 HA 的 SEM 照片

Fig. 3 SEM images of Fe-HA and HA

(a) Co-precipitation Fe-HA; (b) Coprecipitation-hydrothermal Fe-HA; (c) Coprecipitation-hydrothermal HA

所增大。当加入 CTAB 和 PEG 作为分散剂后, HA 的 $R$ 值增大到 0.6 0.7, 定向度明显增加, 说明加入 分散剂改善浆料的分散性后, 磁场对 HA 晶粒的影 响加强, 使其在强磁场中的定向效果得到了加强。

从上述结果看出, 加入分散剂 PEG 的 $R$ 值最 大。共沉淀-水热法 $5 \mathrm{~mol} \% \mathrm{Fe}-\mathrm{HA}(\mathrm{A})$ 、共沉淀-水热 法 HA(B) 和共沉淀法 $5 \mathrm{~mol} \% \mathrm{Fe}-\mathrm{HA}(\mathrm{C})$ 三种粉体均使 用 $5 \mathrm{wt} \% \mathrm{PEG}$ 作分散剂配浆, 在强磁场中定向的 XRD 结果如图 6 所示。其中共沉淀-水热法 HA 浆 料在强磁场中定向, 经计算 $R=0.23$, 小于普通 $\mathrm{HA}$ 的 $R$ 值; 共沉淀-水热法 $5 \mathrm{~mol} \% \mathrm{Fe}-\mathrm{HA}$ 浆料在强磁 场中定向, 经计算 $R=0.65$, 大于普通 $\mathrm{HA}$ 的 $R$ 值; 共


图 4 不同合成方法的 HA 和 Fe-HA 的 TEM 照片

Fig. 4 TEM images of Fe-HA and HA prepared with different methods

(a) Co-precipitation HA; (b) Co-precipitation Fe-HA; (c) Coprecipitation-hydrothermal HA; (d) Coprecipitation-hydrothermal Fe-HA

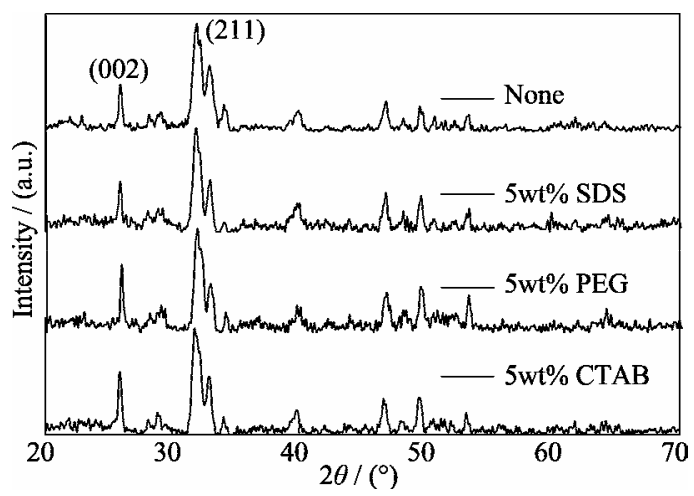

图 5 使用不同分散剂在强磁场中注浆成型的陶瓷片 XRD 图谱

Fig. 5 XRD patterns of the samples with different dispersant casting in high magnetic field

沉淀法 5mol\%Fe-HA 浆料在强磁场中定向, 经计算 $R=0.33$ ，与普通 $\mathrm{HA}$ 的 $R$ 值无异。

Fe-HA 和 HA 在强磁场中的作用原理可以由图 7 简要说明。

晶体在强磁场中的定向原理如下：磁各向异性 晶体各晶轴磁化率不同，因此在强磁场中各晶轴受 到磁化力不同。各晶轴磁化力的差异使得晶体发生 旋转, 直到系统磁化能最低的状态, 形成取向。当晶 体易磁化轴与长轴一致时，在强磁场中长轴会平行 于强磁场; 当晶体易磁化轴与长轴垂直时, 在强磁 场中长轴会垂直于强磁场。

$\mathrm{HA}$ 为抗磁性晶体, 六方晶形, $a 、 b$ 轴为易磁化 轴，因此 HA 的长轴( $c$ 轴)会垂直于强磁场方向进行 


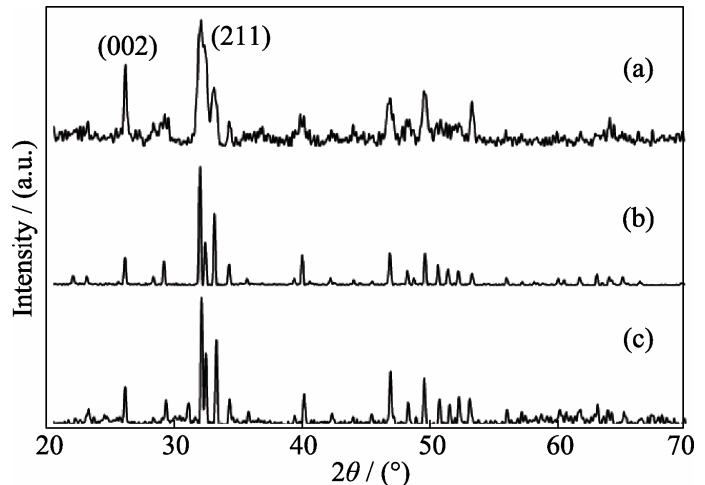

图 6 Fe-HA 和 $\mathrm{HA}$ 在强磁场中注浆成型的陶瓷片 XRD 图谱 Fig. 6 XRD patterns of Fe-HA and HA samples casting in high magnetic field

(a) Coprecipitation-hydrothermal Fe-HA; (b) Coprecipitation-hydrothermal HA; (c) Co-precipitation Fe-HA

(a)

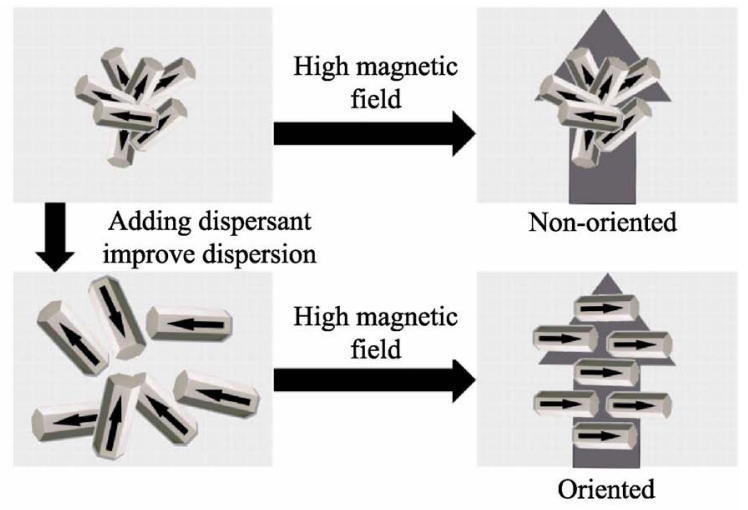

(b)



图 $7 \mathrm{HA} / \mathrm{Fe}-\mathrm{HA}$ 在强磁场中的定向原理示意图

Fig. 7 Orientation principle of HA/Fe-HA in high magnetic field

(a) Effect of dispersant on HA orientation; (b) Effect of Fe ions on the orientation of HA

排布, 并且由于在强磁场垂直面上, 晶体磁各向异 性能均为最低, 因此 $c$ 轴的排列方向在强磁场垂直 面上是无序的，所以不能达到 $c$ 轴的单轴取向。

共沉淀一水热法合成的 HA, 单分散良好, 具备 定向的条件, 在强磁场的作用下, $c$ 轴垂直于强磁场 方向排布, 因此 $R$ 值有所下降, 这与图 6 结果一致。 共沉淀法合成的 Fe-HA 呈针簇状, 而不是单分散的
晶体，每个针簇状结构中的 Fe-HA 晶体分别受到强 磁场的作用，但由于每个晶体的方向不一致，作用 力相互抵消, 因此在强磁场中并不会出现定向效果, $R$ 值也没有发生明显的变化, 如图 7(a)所示。共沉淀 一水热法制得的 Fe-HA, 在垂直方向的强磁场作用 下, $R$ 值不但没有降低反而有所升高, 这说明在 $\mathrm{Fe}$ 离子掺杂改性后, Fe-HA 的 $c$ 轴不再垂直于外磁场 方向排布, 而是平行于外磁场方向排布, 如图 7(b)所 示。这同时说明，通过 Fe 离子掺杂对 HA 顺磁改性， 可能改变了 HA 的易磁化轴, 即 $c$ 轴变为易磁化轴, 使 Fe-HA 可以在单一方向外磁场中定向。

但同时也发现，与理想情况不一致的是，理论 上 HA 在强磁场中 $c$ 轴会垂直于强磁场, 因此理想 状况下, 施加坚直方向强磁场, 其陶瓷表面 $R$ 值应 该为 0 , 但实验结果中, HA 的 $R$ 值在强磁场中并没 有下降到 0 , 这说明 $\mathrm{HA}$ 在强磁场中的定向并不完 全，有可能是强磁场强度不够大或者浆料的分散性 不够好导致的，这同样也有可能是造成共沉淀一水 热法制备的 Fe-HA 在强磁场中定向不完全的原因。

陶瓷表面纳米结构具有诱导成骨的作用，构建 定向排列纳米晶陶瓷有助于进一步精细调控生物陶 瓷表面的微纳米结构, 设计生物活性骨修复材料。 此外, 陶瓷表面的定向纳米结构可用于选择性吸附 细胞蛋白质, 对于调控材料的生物学性能及用于分 析诊断有潜在的应用前景。

\section{3 结论}

1) HA 在合成过程中加入铁离子改性，可以使 其由原本的抗磁性转变为顺磁性。

2) 共沉淀法 Fe-HA 微观形貌为针簇状, 这种 非单分散的针簇状结构在强磁场中不能定向; 共沉 淀一水热法 Fe-HA 微观形貌为短棒状, 单分散性良 好, 在强磁场中有定向效果。

3) 配制浆料时, 选择合适的分散剂改善浆料 的分散性能够一定程度上加强定向效果。

4) Fe 掺杂改性前, $c$ 轴不是 HA 的易磁化轴, HA 在强磁场中不能单轴定向而发生取向; 改性后, Fe-HA 在单一方向强磁场中能够在一定程度上沿 $c$ 轴取向, $c$ 轴可能变为 Fe-HA 的易磁化轴, 说明采用 Fe 掺杂是制备有取向性 HA 晶体结构的生物陶瓷的 有效方法之一。

\section{参考文献:}

[1] LIN K L, WU C T, CHANG J. Advances in synthesis of calcium phosphate crystals with controlled size and shape. Acta Biomateri- 
alia, 2014, 10(10): 4071-4102.

[2] LIN K L, XIA L G, GAN J B, et al. Tailoring the nanostructured surfaces of hydroxyapatite bioceramics to promote protein adsorption, osteoblast growth, and osteogenic differentiation. ACS Applied Materials \& Interfaces, 2013, 5(16): 8008-8017.

[3] XIA L G, LIN K L, JIANG X Q, et al. Effect of nano-structured bioceramic surface on osteogenic differentiation of adipose derived stem cells. Biomaterials, 2014, 35(30): 8514-8527.

[4] LIU C L, ZHAI H L, ZHANG Z S, et al. Cells recognize and prefer bone-like hydroxyapatite: biochemical understanding of ultrathin mineral platelets in bone. ACS Applied Materials \& Interfaces, 2016, 8(44): 29997-30004.

[5] LI S Q, WU C Y, SASSA K, et al. The control of crystal orientation in ceramics by imposition of a high magnetic field. Materials Science and Engineering a-Structural Materials Properties Microstructure and Processing, 2006, 422(1/2): 227-231.

[6] AKIYAMA J, HASHIMOTO M, TAKADAMA H, et al. Formation of c-axis aligned polycrystal hydroxyapatite using high magnetic field with mechanical sample rotation. Materials Transactions, 2005, 46(2): 203-206.

[7] AKIYAMA J, HASHIMOTO M, TAKADAMA H, et al. Orientation of hydroxyapatite $c$-axis under high magnetic field with mold rotation and subsequent sintering process. Materials Transactions, 2005, 46(11): 2514-2517.

[8] CHANDRA V S, BASKAR G, SUGANTHI R V, et al. Blood compatibility of iron-doped nanosize hydroxyapatite and its drug release. ACS Applied Materials \& Interfaces, 2012, 4(3): 1200-1210.

[9] KANCHANA P, LAVANYA N, SEKAR C. Development of amperometric L-tyrosine sensor based on Fe-doped hydroxyapatite nanoparticles. Materials Science \& Engineering C-Materials for Biological Applications, 2014, 35: 85-91.

[10] SATO M, NAKAHIRA A. Influence of Fe addition to hydroxyapatite by hydrothermal process. Journal of the Ceramic Society of Japan, 2013, 121(1415): 559-562.

[11] KRAMER E R, MOREY A M, STARUCH M, et al. Synthesis and characterization of iron-substituted hydroxyapatite via a simple ion-exchange procedure. Journal of Materials Science, 2013, 48(2): 665-673.

[12] TAMPIERI A, D'ALESSANDRO T, SANDRI M, et al. Intrinsic magnetism and hyperthermia in bioactive Fe-doped hydroxyapatite. Acta Biomaterialia, 2012, 8(2): 843-851.

[13] LIU X G, LIN K L, WU C T, et al. Multilevel hierarchically ordered artificial biomineral. Small, 2014, 10(1): 152-159.

[14] LI L, MAO C Y, WANG J M, et al. Bio-inspired enamel repair via Glu-directed assembly of apatite nanoparticles: an approach to biomaterials with optimal characteristics. Advanced Materials, 2011, 23(40): 4695-4701.

[15] FAN Y, SUN Z, MORADIAN-OLDAK J. Controlled remineralization of enamel in the presence of amelogenin and fluoride. Biomaterials, 2009, 30(4): 478-483. 